\title{
Radiographic and anatomic investigation on the prevalence of bifid mandibular canals in cone beam computed tomography scans
}

\author{
Caroline Casagrande ${ }^{1}$, Sther Garcia Orestes ${ }^{1}$, Angela Deliga Schroder ${ }^{2}$, Antonio Adilson Soares \\ de Lima $^{1}$, Ademir Franco ${ }^{3}$, Irina Makeeva ${ }^{3}$, Ângela Fernandes ${ }^{1}$ \\ ${ }^{1}$ Federal University of Paraná, Paraná,Brazil \\ ${ }^{2}$ School of Health Sciences, Pontifícia Universidade Católica do Paraná, Paraná, Brazil \\ ${ }^{3}$ Institute of Dentistry, Sechenov University, Moskow, Russia
}

\section{Abstract}

Background. Knowing the radiologic anatomy of the mandibular canal is essential to perform optimal anesthetic techniques in dentistry, especially in endodontic and surgical procedures. Bifid mandibular canals figure as anatomic variations that may hamper dental anesthesia and lead to surgical accidents.

Objective. This study aims to investigate the prevalence of bifid mandibular canals in cone beam computed tomography (CBCT) images of patients under dental treatment.

Material and methods. The sample consisted of 700 patients (448 females and 252 males) under dental treatment. CBCT images taken from each patient for dental treatment purposes were analyzed retrospectively in order to search for bifid mandibular canals. The detected bifid mandibular canals were classified according to their morphological arrangement and the prevalence of this anatomic variation was tested for association with patients' sex using Pearson's Chi-square test $\left(\chi^{2}\right)$.

Results. The prevalence rate of bifid mandibular canals reached $2 \%(n=14)$. Mandibular canals with an additional bifurcation towards the anterior region of the mandibular ramus were the most prevalent (100\%). Lack of statistical association was observed between the occurrence of bifid mandibular canals and patients' sex $(p>0.05)$.

Conclusion. Despite uncommon, bifid mandibular canals may occur. Dentists must be aware of this anatomic variation to perform safely and optimally in the clinical routine.

Key words: anatomy, cone beam computed tomography, mandibular canal, radiology, stomatology.

For citation: Casagrande C., Orestes S.G., Schroder A.D., Soares de Lima A.A., Ademir Franco A., Makeeva I., Fernandes A. Radiographic and anatomic investigation on the prevalence of bifid mandibular canals in cone beam computed tomography scans. Sechenovsky vestnik. 2018; 3 (33): 54-58.

\section{CONTACT INFORMATION:}

Caroline Casagrande, General Dentist, Department of Stomatology, Federal University of Paraná

Address: Avenida Sete de Setembro 3165 - Térreo Curitiba, Paraná, Brazil

Tel.: +(55) 41987183030

E-mail: franco.gat@gmail.com

The article received: June 9, 2018

The article approved for publication: September 3, 2018

\section{INTRODUCTION}

Radiographic assessment of the anatomy of dentomaxillofacial structures is fundamental to guide the best practices at the dental clinic. Anesthetic procedures are necessary prior to most of the dental treatments, especially for root canal therapeutics in Endodontics and any surgical interventions in Periodontology and Stomatology [1-3]. Success and effectiveness of anesthetic procedures rely on the selection of the anesthetic drug, technique and knowledge of craniofacial anatomy [4].
The most common anesthetic techniques for therapeutic interventions in the mandible aim to reach and block the Inferior Alveolar Nerve (IAN) [5]. Following an intraosseous pathway together with a vascular bundle, this nerve provides sensitive response to the mandibular teeth and adjacent soft tissue - including the lower lip [6]. The intraosseous pathway that protects the IAN and guides it through the mandible is named mandibular canal [7]. This anatomic structure is distributed bilaterally in the mandible and extends from the mandibular foramen to the mental foramen [7]. 
In the dental practice, the mandibular canal is often examined with the aid of panoramic radiographs. However, especial clinical procedures may require a deeper anatomical analysis of the mandibular canal, such complex extractions of impacted or unerupted third molars [8] and case-specific dental implant placement [9]. In the first, a high risk of neurovascular injuries is faced when the mandibular canal has a close relation with the third molar [10], while in the second injuries may be expected from the iatrogenic trauma between the neurovascular bundle and the anesthetic needle, bone drill or the implant itself [11].

In order to perform safely and optimally, cone beam computed tomography (CBCT) may be used to have a better view of the anatomy of the mandibular canal [12]. This modality of imaging exam contributes not only to anesthetic procedures but also to the surgical intervention itself. In opposite, anesthetic and surgical procedures may be hampered due to potential anatomic variations in the mandibular canal. Bifid mandibular canals were previously reported in the scientific literature [13]. This variation consists of a bifurcation in the mandibular canal that is detectable through imaging exams and must be considered prior to any intervention in the mandible. Knowing the prevalence of this anatomic alteration and the correct interpretation of imaginological exams are necessary to carry evidence-based practices in the dental routine.

This study aimed to perform a radiographic and anatomic assessment on the prevalence of bifid mandibular canals in CBCT scans of patients that underwent dental treatment and test the potential association of these findings with their sex.

\section{MATERIAL AND METHODS Ethical aspects and research design}

The present study was carried with the approval of the local Committee of Ethics in Human Research under the protocol numbers 907.032.10.03 and 0019.0.091.000-10. The study was designed as observational, cross-sectional and retrospective and was performed by experienced researchers in the field of Radiology, Stomatology and Anatomy.

\section{Sampling}

The sample consisted of 700 patients (448 females and 252 males) with age between 6 and 82 years (mean age 32.6 years) that underwent CBCT scanning for dental treatment purposes. The Scanning process was performed with the iCAT imaging device (Imaging Science International, Hatfield, PA, USA). The CBCT device was set with a field of view of $1613 \mathrm{~cm}$, voxel size of $0.25,26.7$ seconds of acquisition time, $37.07 \mathrm{~mA}$ and $120 \mathrm{kVp}$. Slice thickness was set in $0.25 \mathrm{~mm}$ with $1 \mathrm{~mm}$ of distance between slices.

The final sample of patients derived from inclusion and exclusion criteria previously applied. The inclusion criteria consisted of selecting patients treated in the local Department of Stomatology and with no evident radiographic signs of bone deformities or lesions in the mandible. According to the exclusion criteria, patients with not record of their sex in the medical file were not sampled as well patients with poor-quality CBCT scans.

\section{Data extraction}

Digital files of the CBCT scans formatted in Digital Image Communication in Medicine (DICOM) were imported in a personal computer (Dell Inspiron Small Desktop, Dell Technol., Round Rock, TX, USA) equipped with proper monitor (Dell LCL LED 18.5", Dell Technol., Round Rock, TX, USA) for data extraction and analysis.

Using a CBCT imaging navigation software package (VisionQ, Imaging Science International, Hatfield, PA, USA) the process of image analysis was performed. Axial, sagittal and coronal slices (multiplanar view) of the mandible and panoramic reconstructions of each patient were examined in order to observe and register the anatomic arrangement of the mandibular canal. Right and left mandibular canals were classified separately as bifid or not based on their anatomy. For an optimal judgment of the canal bifurcation, the classification described by Langlais et al. [14] (1985) was consulted. According to this classification, bifid mandibular canals may be divided into types I, II, III and IV. Type I consists of an arm-shaped bifurcation from the mandibular foramen to the anterior limit of the mandibular ramus (adjacent to the third molar); type II shows a bifurcation that returns to the main canal forming an island-shaped bone region surrounded by the canals; type III is the manifestation of types I and II in the same patient (bilateral occurrence); type IV consists of a main canal that has origin in two different mandibular foramen (figure 1).

\section{Data analysis}

The obtained data was digitally registered in spreadsheets (Excel, Microsoft Corp., Redmond, WA, USA) and underwent descriptive statistics to verify the prevalence of bifid mandibular canals in the sampled CBCT scans. Next, with the hypothesis that no association would be detected, Pearson's Chi-square $\left(\chi^{2}\right)$ test was used to associate the presence of bifid mandibular canals and patient's sex. Data analysis was performed with SPSS 20.0 software package (IBM Corp., Armonk, NY, USA) for Windows operating system (Microsoft Corp., Redmond, WA, USA). The level of statistical significance was set in $0.05(5 \%)$.

\section{RESULTS}

Out of the 700 CBCT scans of the patients sampled in this study fourteen presented bifid mandibular canals, out of which seven were females and seven were males. This number of patients represented $2 \%$ of the sample (table 1). All the bifid mandibular canals detected in this 


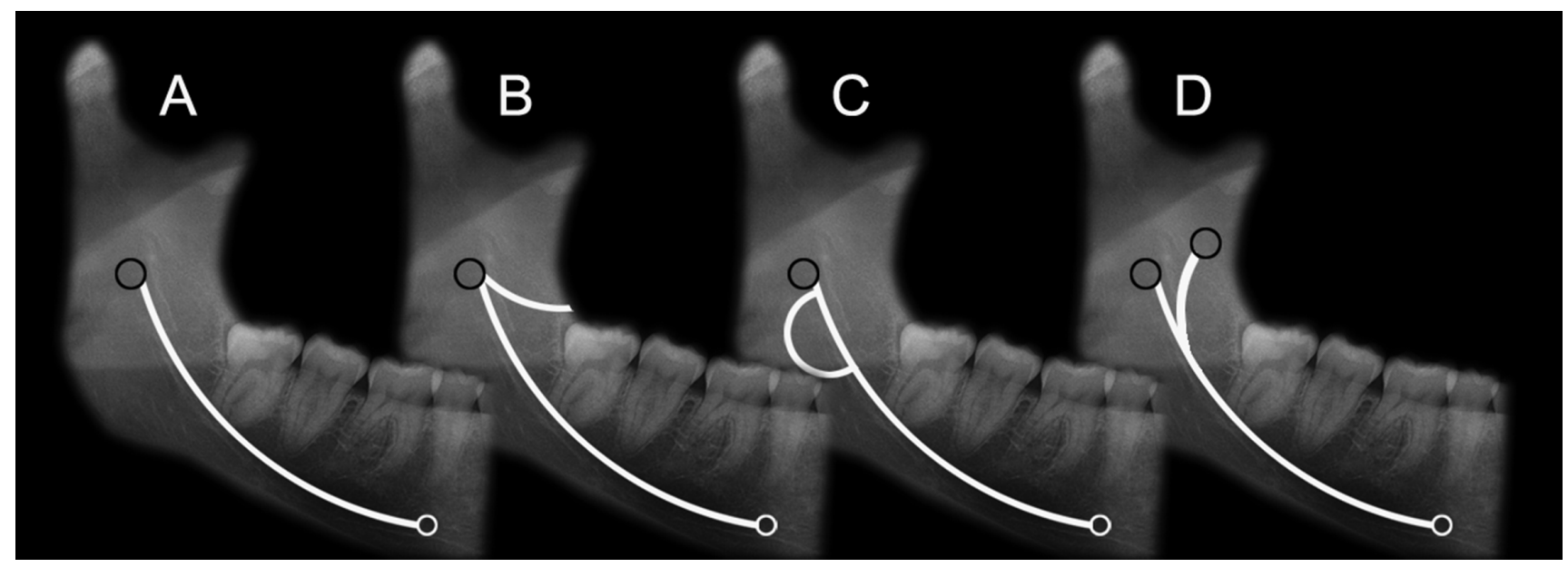

FIGURE 1. Types of bifid mandibular canals according to the classification of Langlais et al. [14] (1985).

Legend: Non-bifid mandibular canal (A) and bifid mandibular canals (B and C). In type I bifid mandibular canals an additional canal is projected from the mandibular foramen to the anterior region of the mandibular ramus (B); In type II an additional canal is formed and returns to the main canal forming an island-shaped bone area (C); in type III there is combination of types I and II in the same patient; in type IV the main canal is originated from two distinct mandibular foramina (D).

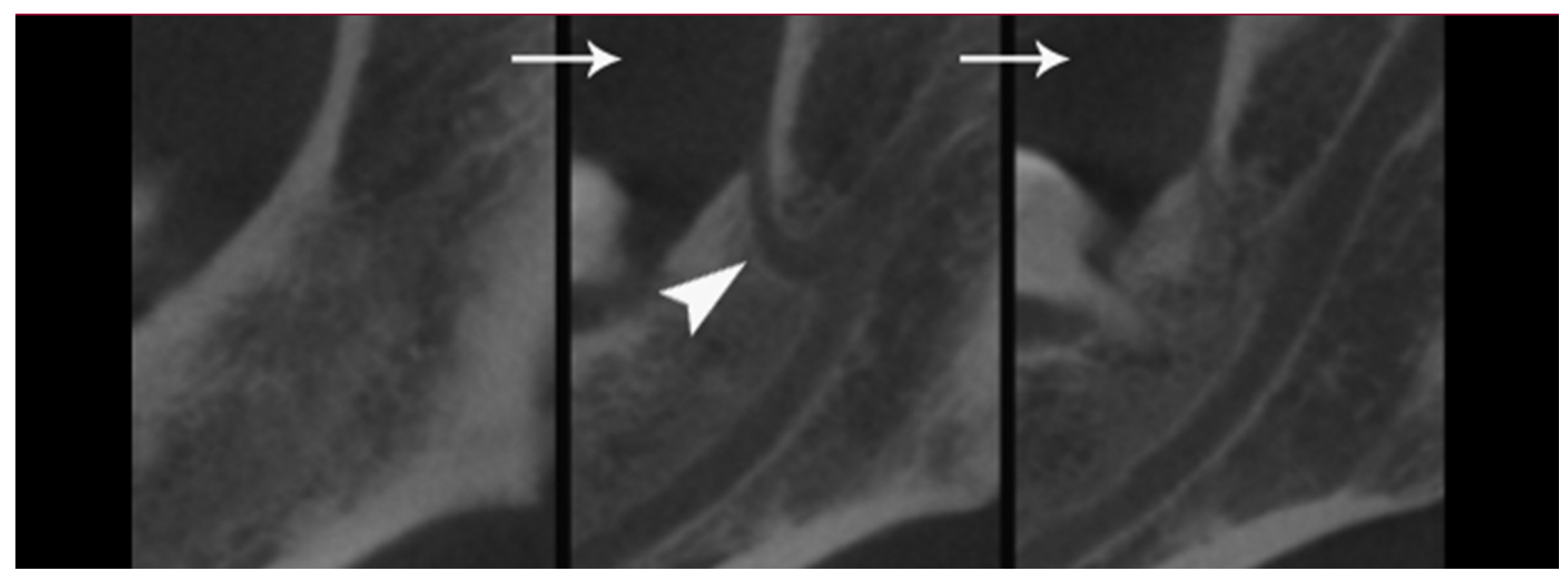

FIGURE 2. Sequential cone beam computed tomography slices (arrows) of a patient with bifid mandibular canal sampled in this study

Legend: Type I bifid mandibular canal (arrow head) extending from the mandibular foramen towards to the anterior region of the mandibular ramus.

study were classified as type I (figure 2) according to Langlais et al. [14] (1985).

In females 3 bifid mandibular canals were observed in the right side, while four were observed in the left side. Oppositely, in males bifid mandibular canals were observed 4 times in the right side and 3 in the right side (table 1).

Pearson's Chi-square statistical test revealed the lack of significant association $(p=0.27)$ between the presence of bifid mandibular canals and sex (table 1).

\section{DISCUSSION}

Knowing the radiographic anatomy of the mandibular canal is a fundamental step to perform surgical procedures in the routine of Stomatology. Invasive techniques in the mandible may trespass the anatomic limits of the mandibular canal and promote injuries to the neurovascular bundle that provides sensitive response and blood supply to the mandibular soft and hard tissues [15]. Bifid mandibular canals may represent a risky condition to surgical procedures. In order to avoid malpractice, Dentists must predict accidents and complications with proper imaginological treatment planning. In this scenario, the present study aimed to investigate the prevalence of bifid mandibular canals in dental patients through CBCT scanning.

The prevalence rate detected in this study reached $2 \%$. Highly similar findings were observed in the scientific literature. Kuczynski et al. [16] (2014) examined 3024 


\section{Table 1}

Sample distribution based on sex, prevalence of bifid mandibular canals and side of occurrence.

\begin{tabular}{lcccccc}
\hline \multirow{2}{*}{ Sex } & \multirow{2}{*}{ Sample $\mathbf{n}(\%)$} & \multicolumn{2}{c}{ Mandibular canal } & \multicolumn{2}{c}{ Side of occurrence } & \multirow{2}{*}{$\boldsymbol{p}$} \\
\cline { 3 - 5 } & & Normal $\mathbf{n}(\%)$ & Bifid $\mathbf{n}(\%)$ & Left $\mathbf{n}(\%)$ & Right $\mathbf{n}(\%)$ & \\
\hline Female & $448(64)$ & $441(63)$ & $7(1)$ & $4(57.14)$ & $3(42.80)$ & 0.27 \\
\hline Male & $252(36)$ & $245(35)$ & $7(1)$ & $3(42.80)$ & $4(57.14)$ & \\
\hline Total & $700(100)$ & $686(98)$ & $14(2)$ & $7(1)$ & $7(1)$ \\
\hline
\end{tabular}

$\mathrm{n}$ : absolute values; \%: relative values; $p$ : statistical significance set at $5 \%$.

patients and observed a prevalence rate of bifid mandibular canals of $1.98 \%(n=60)$. Differently to the present study, these authors performed their study in panoramic radiographs and with a sample considerably larger. In the present study, CBCT was used as an attempt to perform a more accurate assessment of the anatomy of mandibular canals. Despite the differences between studies, the outcomes were nearly the same. It is important to note that these studies were similar in outcomes however the scientific literature reports a large range of prevalence rates of bifid mandibular canals that may vary from $0.2 \%$ to $65 \%[14,17,18]$. In practice, the uncertainty on the exact prevalence rate of this anatomic variation must be interpreted towards more careful treatment planning because. In other words, bifid mandibular canals may not be rare and the Dentists must be aware and prepared to perform effective anesthetic and surgical procedures in these patients.

In a deeper look in the bifid mandibular canals, the present study observed that all the cases were classified as bifurcation type I $(n=14)$. Other types of bifurcations were previously reported in scientific studies. In a study with 1500 panoramic radiographs, Andrade et al. [19] (2014) detected 80 bifid mandibular canals, out of which types I, II, II and IV accounted 51, 26, 2 and 1 cases, respectively. Similarly, Kuczynski et al. [15] (2014) detected 50 bifid mandibular canals type I and 10 type II. These outcomes show a potential prevalence of bifid mandibular canals type I over the other types. Type I bifurcation consists of the projection of the additional mandibular canal to the anterior part of the mandibular ramus - adjacent to the third molar region. In practice, this type of mandibular canal may be interpreted as a risk factor for neurovascular injuries, especially during third molar extractions. In other study, Kuribayashi et al. [20] (2010) found more prevalent bifurcations type II $(n=40)$, followed by type IV $(n=5)$ and type I $(n=2)$.

\section{REFERENCES}

1. Vieira WA, Paranhos LR, Cericato $G O$ et al. Is mepivacaine as effective as lidocaine during inferior alveolar nerve blocks in patients with symptomatic irreversible pulpitis? A systematic review and meta-analysis. Int Endod J 2018 [ahead of print]. DOI: 10.1111/iej.12926

2. Pasquali JG, Narazaki ND, Franco A et al. Assessing the radiographic position of the mental forament in a Braizlian population. J Dent Indonesia 2017; 4 (1): 19-22.
Type II bifurcations include an additional canal that bifurcates and returns to the original canal forming an island-shape area in the mandible. The bifurcation may be restricted to the mandibular ramus or may extend to the mandible body. The last is more risky from a clinical scope because it may represent a complex condition for surgical implant placement along the mandible body. In these cases, CBCT exams may be useful to guide optimal preoperative treatment planning.

The last topic investigated in this study was the potential association between bifid mandibular canals and patients' sex. As expected according to the research hypothesis, no association was found between both variables. In practice, it suggests that any patient may reveal bifid mandibular canals and these canals must be radiographically assessed prior to clinical decisions.

The prevalence rate of bifid mandibular canals expressed in this investigation was compatible to most of the scientific literature. However, exact values are still uncertain. Future studies should be designed to systematically review the current evidences in science in order to enable more solid and well-supported conclusions. With a large sample of CBCT scans, the present study contributes to the scientific community by adding more information on the potential prevalence rates of bifid mandibular canals.

\section{CONCLUSION}

A prevalence rate of bifid mandibular canals of $2 \%$ was detected in this study; all the cases involved type I bifurcations; and no association was detected between the occurrence of bifid mandibular canals and patients' sex.

Dentists must be aware of potential anatomic variations in the mandibular canals, such as bifurcations. CBCT imaging exams figure as tools able to guide the best clinical practices, especially to plan surgical interventions (e.g. third molar extraction and dental implant placement) in patients with bifid mandibular canals.

3. Pereira $P N$, Fernandes A, Gugisch $R C$ et al. Radiographic assessment of the mandibular foramen in children: focus on anesthetic procedures. Arch Oral Res 2013; 9 (3): 279-83.

4. Malamed $S$. Handbook of local anesthesia. $6^{\text {th }}$ ed. St Louis: Elsevier 2013.

5. Khalil $H$. A basic review on the inferior alveolar nerve block techniques. Anesth Essays Res 2014; 8 (1): 3-8. 
6. Alhassani AA, AlGhamdi AST. Inferior alveolar nerve injury in implant deitstry: diagnosis, causes, prevention and management. J Oral Implantol 2010; 36 (5): 401-7.

7. Carter RB, Keen EN. The intramandibular course of the inferior alveolar nerve. J Anat 1971; 108 (3): 433-40.

8. Matzen LH, Wenzel A. Efficacy of CBCT for assessment of impacted mandibular third molars: a review - based on a hierarchical model of evidence. Dentomaxillofac Radiol 2015; 44 (1): 20140189.

9. Jacobs $R$, Salmon B, Codari $M$ et al. Cone beam computed tomography in implant dentistry: recommendations for clinical use. BMC Oral Health 2018; 18: 88.

10. Miclotte A, Franco A, Guerrero MA et al. The association between orthodontic treatment and third molar position, inferior alveolar nerve involvement, and prediction of wisdom tooth eruption. Surg Radiol Anat 2015; 37 (4): 333-9.

11. Joudzbalys $G$, Wang $H L$, Sabalys $G$ et al. Inferior alveolar nerve injury associated with implant surgery. Clin Oral Implant Res 2013; 24: 183-90.

12. Oliveira-Santos C, Capelozza ALA, Dezzoti MSG et al. Visibility of the mandibular canal on CBCT cross-sectional images. J App Oral Sci 2011; 19 (3): 240-3.

13. Wolf KT, Brokaw EJ, Bell A, Joy A. Variant inferior alveolar nerves and implications for local anesthesia. Anesth Prog 2016; 63 (2): 84-90.
14. Langlais RP, Broadus $R$, Glass BJ. Bifid mandibular canals in panoramic radiographs. J Am Dent Assoc 1985; 110 (6): 923-6.

15. Yu SK, Lee MH, Jeon YH et al. Anatomical configuration of the inferior alveolar neurovascular bundle: a histomorphometric analysis. Surg Radiol Anat 2016; 38 (2): 195-201.

16. Kuczynski A, Kucharski W, Franco A et al. Prevalence of bifid mandibular canals in panoramic radiographs. Surg Radiol Anat 2014; 36 (9): 847-50.

17. Bogdán S, Pataky L, Barabás J et al. Atypical courses of the mandibular canal: comparative examination of dry mandibles and X-rays. J Craniofac Surg 2006; 17 (3): 487-91.

18. Naitoh $M$, Nakahara K, Suenaga $Y$ et al. Comparison between cone-beam and multi-slice computed tomography depicting mandibular neurovascular canal structures. Oral Surg Oral Med Oral Pathol Oral Radiol Endod 2010; 109 (1): e25-31.

19. Andrade YDN, Araujo EBJ, Souza LMA, Groppo FC. Analysis of anatomical variations of the mandibular canal found on panoramic radiographs. Rev Odontol Unesp 2015; 44 (1): $31-6$.

20. Kuribayashi A, Watanabe H, Imaizumi A et al. Bifid mandibular canals: cone beam computed tomography evaluation. Dentomaxillofac Radiol 2010; 39 (4): 235-9. 
